\title{
Collocation Method for Fuzzy Volterra Integral Equations of the Second Kind
}

\section{Zahra Alijani and Urve Kangro}

\author{
Institute of Mathematics and Statistic, University of Tartu \\ J. Liivi 2, Tartu, Estonia \\ E-mail(corresp.): urve.kangro@ut.ee \\ E-mail: zahra.alijani@ut.ee
}

Received March 22, 2019; revised December 7, 2019; accepted December 9, 2019

\begin{abstract}
In this paper we consider fuzzy Volterra integral equation of the second kind whose kernel may change sign. We give conditions for smoothness of the upper and lower functions of the solution. For numerical solution we propose the collocation method with two different basis function sets: triangular and rectangular basis. The smoothness results allow us to obtain the convergence rates of the methods. The proposed methods are illustrated by numerical examples, which confirm the theoretical convergence estimates.
\end{abstract}

Keywords: fuzzy Volterra integral equation, smoothness of solution, triangular basis, rectangular basis, collocation method, convergence rate.

AMS Subject Classification: 45D05; 45N05; 65R20.

\section{Introduction}

Integral equations arise in many scientific and engineering problems. A large class of initial and boundary value problems can be converted to Volterra or Fredholm integral equations. The theory of Volterra integral equations is thoroughly considered in a recent monograph by Brunner [6]. Most Volterra integral equations can not be solved analytically. Hence there is need for numerical solution of these equations. Collocation method is widely used for solving integral equations, for treatment of this method for Volterra integral equations see Brunner [5].

Modeling physical problems using integral equations with the exact parameters is often impossible in real problems. To handle this lack of information, one way is to use uncertainty measures such as the fuzzy concept (Zadeh 1965 [18]).

Copyright (c) 2020 The Author(s). Published by VGTU Press

This is an Open Access article distributed under the terms of the Creative Commons Attribution License (http://creativecommons.org/licenses/by/4.0/), which permits unrestricted use, distribution, and reproduction in any medium, provided the original author and source are credited. 
Instead of using deterministic models of integral equations, we can use fuzzy integral equations, where the values of functions may be fuzzy numbers. Hence there is a need to develop mathematical models and numerical procedures that would appropriately treat general fuzzy integral equations and solve them. The topics related to fuzzy integral equations have received particular attention from the research community during the last few decades.

Before discussing fuzzy integral equations and numerical algorithms for solving them, it is necessary to present a brief introduction to fuzzy numbers. Fuzzy functions were introduced by Chang and Zadeh [7]. Later, Dubois and Prade [8] presented an elementary framework for fuzzy calculus based on the extension principle. Alternative approaches were suggested by Goetschel and Voxman [10], Kaleva [11] and others. The concept of integration of fuzzy functions was introduced by Dubois and Prade [8], and investigated by Goetschel and Voxman [10].

Existence and uniqueness of solutions of fuzzy Fredholm integral equations have been considered e.g. in [2,9] and fuzzy Volterra integral equations in $[9,14$, 17]. Smoothness of solutions, to our knowledge, has not been considered before. We prove smoothness results for fuzzy Volterra integral equation in terms of the smoothness of upper and lower functions; this concept differs from being differentiable in the sense of fuzzy functions, but for obtaining convergence rates for numerical methods, smoothness of upper and lower functions is crucial. In some cases the smoothness results can be obtained from the corresponding results for crisp functions, but in the case when the kernel of the integral equation changes sign, it is more complicated. The smoothness results we obtain are in some sense surprising, since when the fuzzy integral equation is converted to a system of ordinary integral equations, the kernels of the crisp equations are, in general (if the kernel of the original integral equation changes sign), not smooth.

Numerical methods for fuzzy Fredholm integral equations are considered in many papers, e.g. $[1,2,4,9]$. In [4] also a convergence rate of $O(h)$ is obtained. Numerical solution of fuzzy Volterra integral equations is considered in $[13,15,16,17]$, but in many cases it is not proved that the approximate solution is a fuzzy function (in some cases it may be trivial, but in other cases it is not true in general). The convergence rates have usually not been considered. Fuzzy Volterra integral equations with changing sign kernels have, to our knowledge, been considered only in [15], but there, only a trivial special case where the sign can only change on horizontal lines is considered, the smoothness of the solution is not proved, and the convergence results are only valid under additional assumptions not mentioned in the paper. There are also a lot of papers which only describe some numerical method for solving fuzzy integral equations and give some numerical examples, but do not provide any analysis at all.

In this paper, we study the collocation method for solving linear fuzzy Volterra integral equations of the second kind. We approximate the solution using triangular and rectangular basis functions with fuzzy coefficients. We prove existence and uniqueness of solution of the approximate equation and show that the approximate solution is also a fuzzy function (this fact is often 
ignored in papers considering numerical solution of fuzzy equations). We do not use higher order splines, because in this case the approximate solution might not be a fuzzy function. We obtain the rate of convergence of the approximate solution to the exact solution.

We also performed numerical experiments to check the theoretical results. We used different discretization parameters and compared the corresponding errors (measured in fuzzy distance). Again, we have not seen any papers about fuzzy integral equations where convergence rates have been checked numerically.

The paper is organized as follows. Section 2 introduces some preliminaries about fuzzy functions necessary in next sections. In the next section we present the fuzzy Volterra integral equations and state a theorem about existence and uniqueness of the fuzzy solution. In Section 4 we describe collocation methods with triangular and rectangular bases for approximately solving the equation and prove existence and uniqueness of the fuzzy approximate solution.

To obtain the convergence rates for the methods we need results about the smoothness of the solution. In Section 5 we give the conditions under which the upper and lower functions of the solution for fixed $r$ are smooth (differentiable, piecewise twice differentiable or continuously twice differentiable). We do not consider fuzzy smoothness, i.e. we do not prove that the derivatives of the solution (in any sense) are fuzzy functions. This is probably not true in general and this is not needed for obtaining the estimates in Section 6. Finally, in Section 7 we provide some numerical examples which demonstrate that the convergence rate for triangular basis is $O\left(h^{2}\right)$; for the rectangular basis it is $O(h)$ in maximum fuzzy distance, but $O\left(h^{2}\right)$ at collocation points.

\section{Preliminaries}

In this section, we review the fundamental notions of fuzzy numbers and fuzzy functions to be used throughout the paper.

Definition 1. [3] A fuzzy number is a mapping $u: \mathbb{R} \rightarrow[0,1]$ such that

1. $u$ is upper semi-continuous;

2. $u(x)=0$ outside some interval $[a, d]$;

3. there are real numbers $b$ and $c, a \leq b \leq c \leq d$, for which

i) $u(x)$ is monotonically increasing on $[a, b]$,

ii) $u(x)$ is monotonically decreasing on $[c, d]$,

iii) $u(x)=1, b \leq x \leq c$.

Fuzzy numbers can also be represented in parametric form as follows.

DEFINITION 2. [3] An arbitrary fuzzy number in parametric form is represented by an ordered pair of functions $(\underline{u}(r), \bar{u}(r)), 0 \leq r \leq 1$, which satisfy the following requirements

1. $\underline{u}(r)$ is a bounded monotonically increasing, left continuous function on $(0,1]$ and right continuous at 0 , 
2. $\bar{u}(r)$ is a bounded monotonically decreasing, left continuous function on $(0,1]$ and right continuous at 0 ,

3. $\underline{u}(r) \leq \bar{u}(r), \quad 0 \leq r \leq 1$.

For arbitrary $u=(\underline{u}(r), \bar{u}(r)), v=(\underline{v}(r), \bar{v}(r))$ and $k \in \mathbb{R}$ we define addition and multiplication by $k$ as $(u+v)(r)=(\underline{u}(r)+\underline{v}(r)), \overline{(u+v)}(r)=(\bar{u}(r)+\bar{v}(r))$, $\underline{k u}(r)=k \underline{u}(r), \overline{k u}(r)=k \bar{u} \overline{(r), \text { if } k} \geq 0, \overline{k u}(r)=k \underline{u}(r), \underline{k u}(r)=k \bar{u}(r)$, if $k<0$.

A crisp (i.e. not fuzzy) number $\alpha$ is simply represented by $\underline{u}(r)=\bar{u}(r)=\alpha$, $0 \leq r \leq 1$. Some special cases of fuzzy numbers are trapezoidal fuzzy numbers, where $\underline{u}(r), \bar{u}(r)$ are linear functions, triangular fuzzy numbers, which are trapezoidal numbers with $\underline{u}(1)=\bar{u}(1)$, and interval numbers, where $\underline{u}(r), \bar{u}(r)$ are constants.

The set of all fuzzy numbers is denoted by $E$. Note that $E$ is not a vector space, because $u+(-u) \neq 0$ in general.

Next we will define the metric $D$ in $E$.

Definition 3. For arbitrary fuzzy numbers $u, v$, we use the distance

$$
D(u, v)=\sup _{0 \leq r \leq 1} \max \{|\bar{u}(r)-\bar{v}(r)|,|\underline{u}(r)-\underline{v}(r)|\} .
$$

It is shown that $(E, D)$ has been a complete metric space [3].

Following Goetschel and Voxman [10] we define the integral of a fuzzy function using the Riemann integral concept.

Definition 4. Let $f:[a, b] \rightarrow E$. For each partition $P=\left\{t_{0}, \ldots, t_{n}\right\}$ of $[a, b]$ and for arbitrary $\xi_{i} \in\left[t_{i-1}, t_{i}\right], 1 \leq i \leq n$ suppose

$$
R_{P}=\sum_{i=1}^{n} f\left(\xi_{i}\right)\left(t_{i}-t_{i-1}\right), \quad \Delta:=\max \left\{t_{i}-t_{i-1}, i=1, \ldots, n\right\} .
$$

The definite integral of $f(t)$ over $[a, b]$ is $\int_{a}^{b} f(t) d t=\lim _{\Delta \rightarrow 0} R_{P}$ provided this limit exists in metric $D$.

If the fuzzy function $f(t)$ is continuous in the metric D, its definite integral exists and

$$
\int_{a}^{b} f(t) d t=\left(\int_{a}^{b} \underline{f}(t, r) d t, \int_{a}^{b} \bar{f}(t, r) d t\right),
$$

where $(f(t, r), \bar{f}(t, r))$ is the parametric form of $f(t)$.

It should be noted that the fuzzy integral can be also defined using the Lebesgue-type approach [11]. Definition of the fuzzy integral using formula (2.1) is more convenient for numerical calculations.

\section{Fuzzy Volterra integral equation}

A fuzzy Volterra integral equation of the second kind is given by

$$
g(t)=f(t)+\int_{0}^{t} K(s, t) g(s) d s, \quad t \in[0, T],
$$


where $K(s, t): D_{T} \rightarrow \mathbb{R}$ is a function called the kernel of the integral equation with domain $D_{T}=\{(s, t) ; 0 \leq s \leq t \leq T\}$ and $f(t)$ is a given fuzzy function of

$t$. If $f(t)$ is a crisp function then equation (3.1) possesses crisp solution and if $f(t)$ is a fuzzy function then the solution is fuzzy.

Existence and uniqueness of solution for fuzzy Volterra integral equations is proved in [14], where the result is given for a nonlinear Volterra integral equation, whose kernel is Lipschitz with respect to the unknown function. Since our equation is linear, this condition is trivially satisfied. In addition, in [14] the existence of the solution is only obtained locally, but in the linear case the existence is global, i.e. in $[0, T]$.

Theorem 1. Let the kernel $K: D_{T} \rightarrow R$ and the fuzzy function $f:[0, T] \rightarrow E$ be continuous. Then equation (3.1) has a unique continuous fuzzy solution on $[0, T]$.

\section{Numerical methods}

Several numerical techniques have been used successfully for fuzzy integral equations $[1,2,4,9,13,15,16,17]$. In many cases it is not proved that the approximate solution is a fuzzy function. Sometimes it follows from the construction, but whenever we have to solve a system of equations to find some unknown coefficients, it is not obvious at all. In this section we discuss in details the collocation method. The idea of collocation methods is the following: we look for solutions in a finite-dimensional approximation space $X_{N}$, where $N$ is an approximation parameter, usually connected with the dimension of the approximation space, and require that the equation is exactly satisfied at some collocation points. Different approximation spaces can be used, usually splines, polynomials or trigonometric polynomials are used. Here we use piecewise linear and piecewise constant splines with triangular and rectangular basis functions correspondingly. In these cases we prove that the approximate solution is always a fuzzy function.

\subsection{Collocation method with triangular basis}

Let $0=t_{0}<t_{1}<\cdots<t_{N}=T$ be a partition of $[0, T]$ and let $h_{k}=t_{k}-t_{k-1}$, $k=1, \ldots, N$.

Definition 5 . The functions $\phi_{0}, \ldots, \phi_{N}$ defined by

$$
\begin{aligned}
& \phi_{0}(t)= \begin{cases}1-\frac{t-t_{0}}{h_{1}}, & t_{0} \leq t \leq t_{1}, \\
0, & \text { otherwise },\end{cases} \\
& \phi_{k}(t)= \begin{cases}\frac{\left(t-t_{k-1}\right)}{h_{k}}, & t_{k-1} \leq t \leq t_{k}, \\
1-\frac{\left(t-t_{k}\right)}{h_{k+1}}, & t_{k} \leq t \leq t_{k+1}, \quad k=1, \ldots, N-1, \\
0, & \text { otherwise },\end{cases}
\end{aligned}
$$




$$
\phi_{N}(t)= \begin{cases}\frac{\left(t-t_{N-1}\right)}{h_{N}}, & t_{N-1} \leq t \leq t_{N} \\ 0, & \text { otherwise }\end{cases}
$$

are called triangular basis functions.

For the collocation points we use the partition points $t_{k}, k=0,1, \ldots, N$. Often the uniform mesh $t_{k}=k h, h=\frac{T}{N}, k=0, \ldots, N$ is used, but sometimes nonuniform grids are useful, especially if the solution is not very smooth near some point. The theory works also for the general case. Let in the following $h=\max _{k=1, \ldots, N} h_{k}$.

We look for solution of equation (3.1) in the form

$$
g_{N}(t)=\sum_{k=0}^{N} c_{k} \phi_{k}(t)
$$

where $\phi_{k}(t)$ are triangular basis functions and $c_{k}, k=0,1, \ldots, N$ are fuzzy numbers. The collocation equations are

$$
g_{N}\left(t_{n}\right)=f\left(t_{n}\right)+\int_{0}^{t_{n}} K\left(s, t_{n}\right) g_{N}(s) d s, n=0, \ldots, N .
$$

Substituting (4.1) into these equations we get

$$
c_{n}=\int_{t_{n-1}}^{t_{n}} c_{n} K\left(s, t_{n}\right) \phi_{n}(s) d s+f\left(t_{n}\right)+\sum_{k=0}^{n-1} \int_{t_{k-1}}^{t_{k+1}} c_{k} K\left(s, t_{n}\right) \phi_{k}(s) d s, n=0, \ldots, N \text {, }
$$

where for simplicity we have denoted $t_{-1}=0$. Note that in general, if the kernel changes sign, one cannot take the fuzzy coefficients $c_{k}$ in front of the integral sign.

We have to solve these linear equations to get the approximate solution. Note that if the coefficients $c_{n}$ are fuzzy numbers then the approximate solution given by (4.1) is a fuzzy function.

\subsection{Collocation method with rectangular basis}

Let $t_{k}, k=0, \ldots, N$ and $h_{k}, k=1, \ldots, N$ be as defined above.

Definition 6. The functions $\psi_{k} k=1, \ldots, N$ defined by

$$
\psi_{k}(t)= \begin{cases}1, & t_{k-1} \leq t \leq t_{k} \\ 0, & \text { otherwise }\end{cases}
$$

are called rectangular basis functions.

In the case of rectangular basis, the best collocation points are the midpoints of the intervals $\tau_{k}=\frac{1}{2}\left(t_{k-1}+t_{k}\right), k=1, \ldots, N$. 
We look for solution of equation (3.1) in the form

$$
g_{N}(t)=\sum_{n=0}^{N} d_{n} \psi_{n}(t)
$$

where $d_{n}, n=1, \ldots, N$ are fuzzy numbers. The collocation equations are

$$
g_{N}\left(\tau_{n}\right)=f\left(\tau_{n}\right)+\int_{0}^{\tau_{n}} K\left(s, \tau_{n}\right) g_{N}(s) d s, n=0, \ldots, N .
$$

Substituting (4.4) into these equations we get

$$
d_{n}=\int_{t_{n-1}}^{\tau_{n}} d_{n} K\left(s, \tau_{n}\right) d s+f\left(\tau_{n}\right)+\sum_{k=1}^{n-1} \int_{t_{k-1}}^{t_{k}} d_{k} K\left(s, \tau_{n}\right) d s, \quad n=1, \ldots, N
$$

Again, if $d_{n}$ are fuzzy numbers then the approximate solution given by (4.4) is a fuzzy function.

\subsection{Existence and uniqueness of the approximate solution}

To show that equations (4.2) and (4.5) have a unique fuzzy solution we use the following lemma.

Lemma 1. Consider the equation

$$
a x=b x-d x+y,
$$

where $a, b$ are crisp coefficients, $y$ is a given fuzzy number, $a>b+d, b, d \geq 0$. Then equation (4.7) has a unique fuzzy solution $x$.

Proof. By converting equation (4.7) to two crisp equations and solving these, we have

$$
\underline{x}=\frac{y(a-b)-d \bar{y}}{(a-b)^{2}-d^{2}}, \quad \bar{x}=\frac{\bar{y}(a-b)-d \underline{y}}{(a-b)^{2}-d^{2}} .
$$

Since $a>b+d, \quad b, d \geq 0$, then $a-b$ and $(a-b)^{2}-d^{2}$ are positive. Also since $\underline{y}$ is nondecreasing (as a function of $r$ ) and $-\bar{y}$ is nondecreasing, we conclude that $\underline{x}$ is nondecreasing. Similarly, since $\bar{y}$ is nonincreasing, $-y$ is nonincreasing and by same reasoning as before we conclude that $\bar{x}$ is nonincreasing. Since $\underline{y}$ and $\bar{y}$ are left continuous, $\underline{x}$ and $\bar{x}$ are left continuous as well. Finally $\underline{x} \leq \bar{x}$, since $\underline{y} \leq \bar{y},-d \bar{y} \leq-d \underline{y}$ and denominators are positive.

Remark 1. In Lemma 1 the condition $b, d \geq 0$ is just a matter of notation. But the assumption $a>b+d$ is necessary: if this is not satisfied, then equation (4.7) does not have a fuzzy solution.

Now by using Lemma 1 we show that the collocation equation (4.2) has a unique approximate fuzzy solution $g_{N}$. 
Theorem 2. Let the kernel $K: D_{T} \rightarrow R$ and the fuzzy function $f:[0, T] \rightarrow E$ be continuous functions. If $h\|K\|_{\infty}<1$ then the equation (4.2) has a unique approximate fuzzy solution $g_{N}$ of the form (4.1).

Proof. We use induction to show that the coefficients $c_{n}$ determined by (4.3) are fuzzy numbers. For $n=0$ equation (4.3) is $c_{0}=f(0)$. Since $f(0)$ is a fuzzy number, $c_{0}$ is also a fuzzy number.

Assume that equation (4.3) has fuzzy solution for $n=0, \ldots, m-1$ and $1 \leq m \leq N$. Let $n=m$, then (4.3) can be written as

$$
\begin{array}{r}
c_{m}=c_{m} \int_{t_{m-1}}^{t_{m}} K_{+}\left(s, t_{m}\right) \phi_{m}(s) d s-c_{m} \int_{t_{m-1}}^{t_{m}} K_{-}\left(s, t_{m}\right) \phi_{m}(s) d s \\
+f\left(t_{m}\right)+\sum_{k=0}^{m-1} \int_{t_{k-1}}^{t_{k+1}} c_{k} K\left(s, t_{m}\right) \phi_{k}(s) d s
\end{array}
$$

where $K_{+}(s, t)=\max \{K(s, t), 0\}$ and $K_{-}(s, t)=\max \{-K(s, t), 0\}$ are the positive and the negative parts of the kernel $K(s, t)$.

By induction assumption we know that

$$
f\left(t_{m}\right)+\sum_{k=0}^{m-1} \int_{t_{k-1}}^{t_{k+1}} c_{k} K\left(s, t_{m}\right) \phi_{k}(s) d s
$$

is a fuzzy number. Hence (4.8) is an equation of form (4.7), where $a=1$,

$$
b=\int_{t_{m-1}}^{t_{m}} K_{+}\left(s, t_{m}\right) \phi_{m}(s) d s, \quad d=\int_{t_{m-1}}^{t_{m}} K_{-}\left(s, t_{m}\right) \phi_{m}(s) d s .
$$

Since $b, d \geq 0$ and for $h$ small enough, $b, d$ are also small enough, we have $a>b+d$ for $h$ small enough. So the assumptions of Lemma 1 are satisfied, therefore there exists a unique fuzzy solution. Since $c_{n}, n=0, \ldots, N$ are fuzzy numbers, the approximate solution (4.1) is a fuzzy function.

A similar result holds for the rectangular basis.

Theorem 3. Let the kernel $K: D_{T} \rightarrow R$ and the fuzzy function $f:[0, T] \rightarrow E$ be continuous functions. If $h\|K\|_{\infty}<1$ then the equation (4.5) has a unique approximate fuzzy solution $g_{N}$ of the form (4.4).

Proof. The proof is similar to the previous theorem.

\section{Smoothness of the solution}

\subsection{Parametric form of the equation}

To prove regularity results and obtain the convergence rates of the collocation method we introduce parametric form of equation $(3.1)$. Let $(\underline{f}(t, r), \bar{f}(t, r))$ 
and $(\underline{g}(t, r), \bar{g}(t, r))$ be parametric forms of $f(t)$ and $g(t)$. Then equation (3.1) is

$$
\underline{g}(t, r)=\underline{f}(t, r)+\int_{0}^{t} \underline{K(s, t) g(t)} d s, \quad \bar{g}(t, r)=\bar{f}(t, r)+\int_{0}^{t} \overline{K(s, t) g(t)} d s .
$$

Denote $K_{+}(s, t)=\max \{K(s, t), 0\}$ and $K_{-}(s, t)=\max \{-K(s, t), 0\}$. Then equation (3.1) can be rewritten as a system of two crisp integral equations

$$
\left\{\begin{array}{l}
\underline{g}(t, r)=\underline{f}(t, r)+\int_{0}^{t}\left(K_{+}(s, t) \underline{g}(s, r)-K_{-}(s, t) \bar{g}(s, r)\right) d s, \\
\bar{g}(t, r)=\bar{f}(t, r)+\int_{0}^{t}\left(K_{+}(s, t) \bar{g}(s, r)-K_{-}(s, t) \underline{g}(s, r)\right) d s .
\end{array}\right.
$$

We define the operators $\mathcal{K}_{+}, \mathcal{K}_{-}: C[0, T] \rightarrow C[0, T]$ by

$$
\left(\mathcal{K}_{+} y\right)(t)=\int_{0}^{t} K_{+}(s, t) y(s) d s, \quad\left(\mathcal{K}_{-} y\right)(t)=\int_{0}^{t} K_{-}(s, t) y(s) d s .
$$

Then we can rewrite system (5.1) as

$$
\left\{\begin{array}{l}
\underline{g}=\underline{f}+\mathcal{K}_{+} \underline{g}-\mathcal{K}_{-} \bar{g} \\
\bar{g}=\overline{\bar{f}}+\mathcal{K}_{+} \bar{g}-\mathcal{K}_{-} \underline{g}
\end{array}\right.
$$

\subsection{Regularity properties}

To derive the convergence rates of our numerical method, we first need to obtain some regularity results. We have to point out that we do not need fuzzy regularity here, we only need regularity of the crisp functions $\underline{g}(\cdot, r), \bar{g}(\cdot, r)$, where $r$ can be considered as a parameter. So we consider the regularity of solution of the system of integral equations (5.1). It is known that if the kernel and the right hand side of Volterra integral equation of the second kind are in $C^{m}$, then the solution is also in $C^{m}$ (see for example [6]), and this applies also for systems. However, if the kernel of the original integral equation (3.1) changes sign, then in our system (5.1) even for smooth $K$ the kernels $K_{+}$and $K_{-}$are only piecewise continuously differentiable. Still we can prove under quite general assumptions that the solution is at least piecewise twice continuously differentiable, and give some additional conditions under which it is twice continuously differentiable. So in this section we mainly deal with the nontrivial case when the kernel changes sign.

Since we consider $r$ as a parameter and never differentiate with respect to $r$, we use in the following the notation $f^{\prime}, \bar{f}^{\prime}$ for derivatives with respect to $t$. We also skip the parameter $r$ inside the proof.

Theorem 4. Let $K \in C\left(D_{T}\right)$ and $f \in C([0, T] ; E)$ be given. Let $g$ be the solution of (3.1). Assume that $K$ changes sign on continuous lines $s=s_{i}(t)$, $t \in\left[\alpha_{i}, \beta_{i}\right], i=1, \ldots, n$ whose endpoints lie on the lines $s=t, s=0$ or $t=T$. For simplicity assume also that at all intersection points of lines $s=s_{i}(t)$, $s=t, s=0$ and $t=T$ only two of the lines are intersecting. Let $r \in[0,1]$ be fixed. 
1. If $\underline{f}(\cdot, r), \bar{f}(\cdot, r) \in C^{1}[0, T]$ and $\frac{\partial K}{\partial t} \in C\left(D_{T}\right)$, then $\underline{g}(\cdot, r), \bar{g}(\cdot, r) \in$ $C^{1}[0, T]$.

2. If additionally $\underline{f}^{\prime \prime}(\cdot, r), \bar{f}^{\prime \prime}(\cdot, r), \frac{\partial^{2} K}{\partial t^{2}}$ are piecewise continuous, and $t \mapsto$ $K(t, t)$ and $s=s_{i}(t)$ are piecewise continuously differentiable, then $\underline{g}^{\prime \prime}(\cdot, r), \bar{g}^{\prime \prime}(\cdot, r)$ are piecewise continuous.

3. If additionally $\underline{f}(\cdot, r), \bar{f}(\cdot, r) \in C^{2}[0, T], \frac{\partial^{2} K}{\partial t^{2}} \in C\left(D_{T}\right)$, and $s=s_{i}(t)$ and $t \mapsto K(t, t)$ are continuously differentiable and

a) at points, where $s_{i}(t)=t \neq 0$, we have $\frac{d K(t, t)}{d t}=0$ and either $s_{i}^{\prime}(t)=1$ or $\frac{\partial K}{\partial t}(t, t)=0$

b) at points, where $s_{i}(t)=0, t \neq 0$, we have either $s_{i}^{\prime}(t)=0$ or $\frac{\partial K}{\partial t}(t, 0)=0$,

then $\underline{g}(\cdot, r), \bar{g}(\cdot, r) \in C^{2}[0, T]$.

Proof. To establish the regularity of $g, \bar{g}$, we differentiate equations (5.1). We have to examine the regularity of integrals of type

$$
W(t)=\int_{0}^{t} U(s, t) d s,
$$

where $U(s, t)$ is one of $K_{+}(s, t) g(s), K_{+}(s, t) \bar{g}(s), K_{-}(s, t) g(s)$ or $K_{-}(s, t) \bar{g}(s)$. Note that $U \in C\left(D_{T}\right)$, since on lines of sign change of $K$ we have $K(s, t)=0$, but derivatives of $K_{+}$and $K_{-}$have jumps on these lines.

Let $t \in(0, T)$ be fixed. If $t$ does not correspond to any endpoints or intersection points of the lines of sign change, then we can renumber the lines in a neighborhood of $t$ in the order of increasing $s$ and denote $s_{0}(t)=0$ and $s_{n+1}(t)=t$. Assuming $\frac{\partial K}{\partial t} \in C\left(D_{T}\right)$ we can differentiate (5.2):

$$
W^{\prime}(t)=U(t, t)+\int_{0}^{t} \frac{\partial U(s, t)}{\partial t} d s=U(t, t)+\sum_{i=0}^{n} \int_{s_{i}(t)}^{s_{i+1}(t)} \frac{\partial U(s, t)}{\partial t} d s .
$$

If $\frac{\partial K}{\partial t} \in C\left(D_{T}\right)$, then $\frac{\partial U(s, t)}{\partial t}$ is continuous inside all integration regions and the limits of integration are also continuous. So all terms on right hand side are continuous at $t$.

Assuming $K$ is (piecewise) twice differentiable with respect to $t$, we can differentiate (5.3) again:

$$
\begin{aligned}
W^{\prime \prime}(t)=\frac{d U(t, t)}{d t} & +\sum_{i=0}^{n}\left(\left.\frac{\partial U(s, t)}{\partial t}\right|_{s_{i+1}(t)-} s_{i+1}^{\prime}(t)-\left.\frac{\partial U(s, t)}{\partial t}\right|_{s_{i}(t)+} s_{i}^{\prime}(t)\right) \\
& +\sum_{i=0}^{n} \int_{s_{i}(t)}^{s_{i+1}(t)} \frac{\partial^{2} U(s, t)}{\partial t^{2}} d s
\end{aligned}
$$


If $\frac{\partial^{2} K}{\partial t^{2}}$ is (piecewise) continuous, then all terms here are (at least piecewise) continuous at $t$.

If $t=t_{*}$ corresponds to an endpoint or intersection point of the lines of sign change, then we have to consider one-sided limits of $W^{\prime}(t)$ and $W^{\prime \prime}(t)$ as $t \rightarrow t_{*}$. We have three cases (they are not exclusive, so we may have several of them at the same time) as is shown in Figure 1.

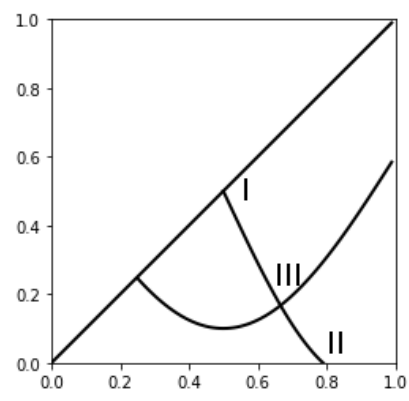

Figure 1. An example of three different intersections of lines of sign change of $K$.

Case I. Lines $s=s_{i}(t)$ and $s=t$ intersect at $t=t_{*}$. We can consider only a small neighborhood of point $\left(t_{*}, t_{*}\right)$, where there are no other lines of sign change. Assume the line $s=s_{i}(t)$ starts at $t=t_{*}$ (if it ends there, the argument is similar). Denote $W_{\varepsilon}(t)=\int_{t_{*}-\varepsilon}^{t} U(s, t) d s$. Then we have

$$
\begin{aligned}
& W_{\varepsilon}^{\prime}(t)=U(t, t)+\int_{t_{*}-\varepsilon}^{t} \frac{\partial U(s, t)}{\partial t} d s \text { for } t<t_{*} \\
& W_{\varepsilon}^{\prime}(t)=U(t, t)+\int_{t_{*}-\varepsilon}^{s_{i}(t)} \frac{\partial U(s, t)}{\partial t} d s+\int_{s_{i}(t)}^{t} \frac{\partial U(s, t)}{\partial t} d s \text { for } t>t_{*} .
\end{aligned}
$$

Since $s_{i}(t) \rightarrow t_{*}$ as $t \rightarrow t_{*}+$, the one-sided limits of $W^{\prime}(t)$ at $t=t_{*}+$ are equal, if $\frac{\partial K}{\partial t} \in C\left(D_{T}\right)$.

Assuming $K$ is (piecewise) twice differentiable with respect to $t$, we have

$$
\begin{aligned}
W_{\varepsilon}^{\prime \prime}(t)= & \frac{d U(t, t)}{d t}+\left.\frac{\partial U(s, t)}{\partial t}\right|_{s=t}+\int_{t_{*}-\varepsilon}^{t} \frac{\partial^{2} U(s, t)}{\partial t^{2}} d s \text { for } t<t_{*}, \\
W_{\varepsilon}^{\prime \prime}(t)= & \frac{d U(t, t)}{d t}+\left.\frac{\partial U(s, t)}{\partial t}\right|_{s_{i}(t)-} s_{i}^{\prime}(t)-\left.\frac{\partial U(s, t)}{\partial t}\right|_{s_{i}(t)+} s_{i}^{\prime}(t) \\
& +\left.\frac{\partial U(s, t)}{\partial t}\right|_{s=t}+\int_{t_{*}-\varepsilon}^{s_{i}(t)} \frac{\partial^{2} U(s, t)}{\partial t^{2}} d s+\int_{s_{i}(t)}^{t} \frac{\partial^{2} U(s, t)}{\partial t^{2}} d s \text { for } t>t_{*} .
\end{aligned}
$$

Now $\frac{U(t, t)}{d t}$ is discontinuous at $t=t_{*}$ in general, unless $\frac{d K(t, t)}{d t}=0$ at $t=t_{*}$. The one-sided limits of the integral terms are equal as $t \rightarrow t_{*}$. The remaining terms give the same limits if $s_{i}^{\prime}(t)=1$ or $\frac{\partial K(s, t)}{\partial t}=0$ at $s=t=t_{*}$.

Case II. Lines $s=s_{i}(t)$ and $s=0$ intersect at $t=t_{*}$. We can consider only a small neighborhood of the point $\left(t_{*}, 0\right)$, where there are no other lines 
of sign change. Denote $W_{\varepsilon}(t)=\int_{0}^{\varepsilon} U(s, t) d s$. Assuming the line $s=s_{i}(t)$ ends at $t=t_{*}$,

$$
\begin{aligned}
& W_{\varepsilon}^{\prime}(t)=\int_{0}^{s_{i}(t)} \frac{\partial U(s, t)}{\partial t} d s+\int_{s_{i}(t)}^{\varepsilon} \frac{\partial U(s, t)}{\partial t} d s \text { for } t<t_{*}, \\
& W_{\varepsilon}^{\prime}(t)=\int_{0}^{\varepsilon} \frac{\partial U(s, t)}{\partial t} d s \text { for } t>t_{*} .
\end{aligned}
$$

Since $s_{i}(t) \rightarrow 0$ as $t \rightarrow t_{*}-$ the one-sided limits of $W_{\varepsilon}^{\prime}(t)$ at $t=t_{*}-$ are equal. For the second derivative we have

$$
\begin{aligned}
W_{\varepsilon}^{\prime \prime}(t)= & \left.\frac{\partial U(s, t)}{\partial t}\right|_{s \rightarrow s_{i}(t)^{-}} s_{i}^{\prime}(t)+\int_{0}^{s_{i}(t)} \frac{\partial^{2} U(s, t)}{\partial t^{2}} d s \\
& -\left.\frac{\partial U(s, t)}{\partial t}\right|_{s \rightarrow s_{i}(t)^{+}} s_{i}^{\prime}(t)+\int_{s_{i}(t)}^{\varepsilon} \frac{\partial^{2} U(s, t)}{\partial t^{2}} d s \text { for } t<t_{*}, \\
W_{\varepsilon}^{\prime \prime}(t)= & \int_{0}^{\varepsilon} \frac{\partial^{2} U(s, t)}{\partial t^{2}} d s \text { for } t>t_{*} .
\end{aligned}
$$

If $K$ is piecewise twice differentiable then the one-sided limits of integrals are equal, since $s_{i}(t) \rightarrow 0$ as $t \rightarrow t_{*}^{+}$. The remaining terms give the same limits if $s_{i}^{\prime}(t)=0$ or $\frac{\partial K(s, t)}{\partial t}=0$.

Case III. Lines of sign change intersect at $t=t_{*}$. Denote these lines by $s=s_{i}(t)$ and $s=s_{j}(t)$ so that for $t<t_{*}$ we have $s_{j}(t)<s_{i}(t)$ and for $t>t_{*}$ we have $s_{i}(t)<s_{j}(t)$. Consider only a small neighborhood of point $\left(t_{*}, s_{*}\right)$, where $s_{*}=s_{i}\left(t_{*}\right)=s_{j}\left(t_{*}\right)$.

Denote $W_{\varepsilon}(t)=\int_{s_{*}-\varepsilon}^{s_{*}+\varepsilon} U(s, t) d s$. Then

$$
\begin{array}{ll}
W_{\varepsilon}(t)=\int_{s_{*}-\varepsilon}^{s_{j}(t)} U(s, t) d s+\int_{s_{j}(t)}^{s_{i}(t)} U(s, t) d s+\int_{s_{i}(t)}^{s_{*}+\varepsilon} U(s, t) d s & \text { for } t<t_{*}, \\
W_{\varepsilon}(t)=\int_{s_{*}-\varepsilon}^{s_{i}(t)} U(s, t) d s+\int_{s_{i}(t)}^{s_{j}(t)} U(s, t) d s+\int_{s_{j}(t)}^{s_{*}+\varepsilon} U(s, t) d s & \text { for } t>t_{*} .
\end{array}
$$

If $K$ is differentiable with respect to $t$ we can take the derivative

$$
\begin{aligned}
& W_{\varepsilon}^{\prime}(t)=\int_{s_{*}-\varepsilon}^{s_{j}(t)} \frac{\partial U(s, t)}{\partial t} d s+\int_{s_{j}(t)}^{s_{i}(t)} \frac{\partial U(s, t)}{\partial t} d s+\int_{s_{i}(t)}^{s_{*}+\varepsilon} \frac{\partial U(s, t)}{\partial t} d s \text { for } t<t_{*}, \\
& W_{\varepsilon}^{\prime}(t)=\int_{s_{*}-\varepsilon}^{s_{i}(t)} \frac{\partial U(s, t)}{\partial t} d s+\int_{s_{i}(t)}^{s_{j}(t)} \frac{\partial U(s, t)}{\partial t} d s+\int_{s_{j}(t)}^{s_{*}+\varepsilon} \frac{\partial U(s, t)}{\partial t} d s \text { for } t>t_{*} .
\end{aligned}
$$

Since $\lim _{t \rightarrow t_{*}} s_{i}(t)=\lim _{t \rightarrow t_{*}} s_{j}(t)=s_{*}$, the one-sided limits are equal.

Assuming $K$ is (piecewise) twice differentiable with respect to $t$ we have

$$
W_{\varepsilon}^{\prime \prime}(t)=\int_{s_{*}-\varepsilon}^{s_{j}(t)} \frac{\partial^{2} U(s, t)}{\partial t^{2}} d s+\int_{s_{j}(t)}^{s_{i}(t)} \frac{\partial^{2} U(s, t)}{\partial t^{2}} d s+\int_{s_{i}(t)}^{s_{*}+\varepsilon} \frac{\partial^{2} U(s, t)}{\partial t^{2}} d s
$$




$$
\begin{aligned}
& +\left.\frac{\partial U(s, t)}{\partial t}\right|_{s_{j}(t)^{-}} s_{j}^{\prime}(t)+\left.\frac{\partial U(s, t)}{\partial t}\right|_{s_{i}(t)^{-}} s_{i}^{\prime}(t) \\
& -\left.\frac{\partial U(s, t)}{\partial t}\right|_{s_{j}(t)^{+}} s_{j}^{\prime}(t)-\left.\frac{\partial U(s, t)}{\partial t}\right|_{s_{i}(t)^{+}} s_{i}^{\prime}(t) \quad \text { for } t<t_{*}, \\
W_{\varepsilon}^{\prime \prime}(t)= & \int_{s_{*}-\varepsilon}^{s_{i}(t)} \frac{\partial^{2} U(s, t)}{\partial t^{2}} d s+\int_{s_{i}(t)}^{s_{j}(t)} \frac{\partial^{2} U(s, t)}{\partial t^{2}} d s+\int_{s_{j}(t)}^{s_{*}+\varepsilon} \frac{\partial^{2} U(s, t)}{\partial t^{2}} d s \\
& +\left.\frac{\partial U(s, t)}{\partial t}\right|_{s_{i}(t)^{-}} s_{i}^{\prime}(t)+\left.\frac{\partial U(s, t)}{\partial t}\right|_{s_{j}(t)^{-}} s_{j}^{\prime}(t) \\
& -\left.\frac{\partial U(s, t)}{\partial t}\right|_{s_{i}(t)^{+}} s_{i}^{\prime}(t)-\left.\frac{\partial U(s, t)}{\partial t}\right|_{s_{j}(t)^{+}} s_{j}^{\prime}(t) \quad \text { for } t>t_{*} .
\end{aligned}
$$

Since two lines of sign change of $K$ intersect at $\left(s_{*}, t_{*}\right)$, it must be a saddle point of $K$, hence $\frac{\partial K(s, t)}{\partial t}=0$ at $\left(s_{*}, t_{*}\right)$, therefore all the terms outside the integral approach 0 as $t \rightarrow t_{*}$. The integral terms give the same limits as $t \rightarrow t_{*}$.

The smoothness of the solution depends on the solution of the integral terms, which we just investigated, and the smoothness of $f$. So assuming $f$ is at least as smooth as the integral terms, the proof is completed.

Remark 2. Theorem 4 does not not cover all possible configurations of lines of sign changes of $K$, e.g. the case where three or more lines intersect at one point. Generally the smoothness of the solution can be investigated similarly in these cases. There are also cases when the first derivative of the solution may be discontinuous, e.g. if there is a vertical line of sign change or when the line of sign change is not a graph of a function (turns back).

For obtaining convergence rates for numerical methods we also need uniform boundedness of derivatives of $\underline{g}, \bar{g}$ with respect to $r$.

Lemma 2. Let the assumptions of Theorem 4, except 2., 3. be satisfied. Assume additionally that there exists constant $B$ such that

$$
\left|\underline{f}^{\prime}(t, r)\right| \leq B, \quad\left|\bar{f}^{\prime}(t, r)\right| \leq B \quad \forall t \in[0, T], r \in[0,1] .
$$

Then there exists constant $C$ such that

$$
\left|\underline{g}^{\prime}(t, r)\right| \leq C, \quad\left|\bar{g}^{\prime}(t, r)\right| \leq C \quad \forall t \in[0, T], r \in[0,1] .
$$

Proof. Continuity of $g$ as a fuzzy-valued function follows from Theorem 1 ; this implies uniform boundedness of $\underline{g}, \bar{g}$. Using expressions for derivatives of $g, \bar{g}$ obtained in the proof of Theorem 4 we get an uniform bound for $\underline{g}^{\prime}, \bar{g}^{\prime}$.

Lemma 3. Let the assumptions of Theorem 4, except 3., be satisfied. Assume additionally that there exists constant $B$ such that

$$
\left|\underline{f}^{\prime \prime}(t, r)\right| \leq B, \quad\left|\bar{f}^{\prime \prime}(t, r)\right| \leq B \quad \forall t \in[0, T], r \in[0,1] .
$$

Then there exists constant $C$ such that

$$
\left|\underline{g}^{\prime \prime}(t, r)\right| \leq C, \quad\left|\bar{g}^{\prime \prime}(t, r)\right| \leq C \quad \forall t \in[0, T], r \in[0,1] .
$$


Proof. Using Lemma 2 and expressions for second derivatives of $\underline{g}, \bar{g}$ in the proof of Theorem 4 we obtain an uniform bound for $\underline{g}^{\prime \prime}, \bar{g}^{\prime \prime}$.

\section{Convergence of the collocation method}

\subsection{Parametric form of the approximate equation}

To analyze the convergence we introduce the parametric form of the approximate equation. Consider the case of triangular basis. Let $c_{n}=\left(\underline{c_{n}}, \overline{c_{n}}\right)$. Then equations (4.3) can be written as

$$
\begin{aligned}
& \underline{c_{n}}=\underline{c_{n}}\left(\mathcal{K}_{+}^{n} \phi_{n}\right)\left(t_{n}\right)-\overline{c_{n}}\left(\mathcal{K}_{-}^{n} \phi_{n}\right)\left(t_{n}\right)+\underline{f\left(t_{n}\right)}+\sum_{k=0}^{n-1}\left(\underline{c_{k}}\left(\mathcal{K}_{+} \phi_{k}\right)\left(t_{n}\right)-\overline{c_{k}}\left(\mathcal{K}_{-} \phi_{k}\right)\left(t_{n}\right)\right), \\
& \overline{c_{n}}=\overline{c_{n}}\left(\mathcal{K}_{+}^{n} \phi_{n}\right)\left(t_{n}\right)-\underline{c_{n}}\left(\mathcal{K}_{-}^{n} \phi_{n}\right)\left(t_{n}\right)+\overline{f\left(t_{n}\right)}+\sum_{k=0}^{n-1}\left(\overline{c_{k}}\left(\mathcal{K}_{+} \phi_{k}\right)\left(t_{n}\right)-\underline{c_{k}}\left(\mathcal{K}_{-} \phi_{k}\right)\left(t_{n}\right)\right) .
\end{aligned}
$$

Here

$$
\left(\mathcal{K}_{+}^{n} \phi_{n}\right)\left(t_{n}\right)=\int_{t_{n-1}}^{t_{n}} K_{+}(s, t) \phi_{n}(s) d s,\left(\mathcal{K}_{-}^{n} \phi_{n}\right)\left(t_{n}\right)=\int_{t_{n-1}}^{t_{n}} K_{-}(s, t) \phi_{n}(s) d s
$$

for $n=1, \ldots, N$. For $n=0$ we can define $\left(\mathcal{K}_{+}^{0} \phi_{0}\right)\left(t_{0}\right)=0,\left(\mathcal{K}_{-}^{0} \phi_{0}\right)\left(t_{0}\right)=0$.

In the case of rectangular basis denote $d_{n}=\left(\underline{d_{n}}, \overline{d_{n}}\right)$. Then the parametric form of equation (4.6) is

$$
\begin{aligned}
& \underline{d_{n}}=\underline{d_{n}}\left(\mathcal{K}_{+}^{n} \psi_{n}\right)\left(\tau_{n}\right)-\overline{d_{n}}\left(\mathcal{K}_{-}^{n} \psi_{n}\right)\left(\tau_{n}\right)+\underline{f\left(\tau_{n}\right)}+\sum_{k=1}^{n-1}\left(\underline{d_{k}}\left(\mathcal{K}_{+} \psi_{k}\right)\left(\tau_{n}\right)-\overline{d_{k}}\left(\mathcal{K}_{-} \psi_{k}\right)\left(\tau_{n}\right)\right), \\
& \overline{d_{n}}=\overline{d_{n}}\left(\mathcal{K}_{+}^{n} \psi_{n}\right)\left(\tau_{n}\right)-\underline{d_{n}}\left(\mathcal{K}_{-}^{n} \psi_{n}\right)\left(\tau_{n}\right)+\overline{f\left(\tau_{n}\right)}+\sum_{k=1}^{n-1}\left(\overline{d_{k}}\left(\mathcal{K}_{+} \psi_{k}\right)\left(\tau_{n}\right)-\underline{d_{k}}\left(\mathcal{K}_{-} \psi_{k}\right)\left(\tau_{n}\right)\right),
\end{aligned}
$$

where

$$
\left(\mathcal{K}_{+}^{n} \psi_{n}\right)\left(\tau_{n}\right)=\int_{t_{n-1}}^{\tau_{n}} K_{+}(s, t) \psi_{n}(s) d s,\left(\mathcal{K}_{-}^{n} \psi_{n}\right)\left(\tau_{n}\right)=\int_{t_{n-1}}^{\tau_{n}} K_{-}(s, t) \psi_{n}(s) d s .
$$

\subsection{Convergence}

To prove the convergence of these methods with triangular and rectangular basis, we use Theorem 13.10 from [12].

Theorem 5. Let $X$ be a Banach space and $X_{N} \subset X$ be a sequence of subspaces. Let $P_{N}: X \rightarrow X_{N}$ be projection operators. Assume that $A: X \rightarrow X$ is a compact linear operator and $I-A$ is injective. Assume that the projectors $P_{N}: X \rightarrow X_{N}$ satisfy

$$
\left\|P_{N} A-A\right\| \rightarrow 0, N \rightarrow \infty .
$$


Then, for sufficiently large $N$, the approximate equation

$$
u_{N}-P_{N} A u_{N}=P_{N} f
$$

is uniquely solvable for all $f \in X$ and there holds an error estimate

$$
\left\|u_{N}-u\right\| \leq M\left\|P_{N} u-u\right\|,
$$

where $u$ is the solution of $u-A u=f$ and the constant $M$ depends only on $A$.

Let $X=C[0, T] \times C[0, T]$,

$$
A=\left(\begin{array}{cc}
\mathcal{K}_{+} & -\mathcal{K}_{-} \\
-\mathcal{K}_{-} & \mathcal{K}_{+}
\end{array}\right), \quad u=\left(\begin{array}{c}
g(\cdot, r) \\
\bar{g}(\cdot, r)
\end{array}\right) \text { for } r \text { fixed }
$$

It is known that $A$ is compact and $I-A$ is injective (see Theorem 1.2.8 in [6]). For triangular basis we define $X_{N}=\operatorname{span}\left\{\phi_{n}, n=0, \ldots, N\right\}$ and $P_{N}$ is then the interpolation projector onto $X_{N}$.

We use the standard estimate for $\left\|P_{N} u-u\right\|_{\infty}$ (e.g. Theorem 11.3 in [12]).

Lemma 4. If $v \in W^{2, \infty}(0, T)$, then for the error in piecewise linear interpolation there holds

$$
\left\|P_{N} v-v\right\|_{\infty} \leq \frac{1}{8} h^{2}\left\|v^{\prime \prime}\right\|_{\infty}
$$

Using Theorem 5 and Lemma 4 we get the error estimate for triangular basis as follows.

Theorem 6. Let $K \in C\left(D_{T}\right), f \in C([0, T] ; E)$. Assume $h \rightarrow 0$ as $N \rightarrow \infty$. Then for sufficiently large $N$ the approximate equation (4.2) has a unique solution $g_{N}$, which converges uniformly to the exact solution $g$ of equation (3.1). If the assumptions of Lemma 3 are satisfied then the error estimate

$$
\sup _{t \in[0, T]} D\left(g_{N}(t), g(t)\right) \leq M h^{2}
$$

holds, where $M$ is a constant not depending on $N$.

Proof. Let $r \in[0,1]$ be fixed. Since $A u \in X$, we have $\left\|P_{N} A u-A u\right\|_{\infty} \rightarrow 0$ as $N \rightarrow \infty$ for all $u \in X$. Since for compact operators, the pointwise convergence implies convergence in norm, we get

$$
\left\|P_{N} A-A\right\|_{\infty} \rightarrow 0 \text { as } N \rightarrow \infty .
$$

By Theorem 5 we get the error estimate

$$
\begin{aligned}
& \left\|\bar{g}_{N}(\cdot, r)-\bar{g}(\cdot, r)\right\|_{\infty} \leq M\left\|P_{N} \bar{g}(\cdot, r)-\bar{g}(\cdot, r)\right\|_{\infty}, \\
& \left\|\underline{g}_{N}(\cdot, r)-\underline{g}(\cdot, r)\right\|_{\infty} \leq M\left\|P_{N} \underline{g}(\cdot, r)-\underline{g}(\cdot, r)\right\|_{\infty},
\end{aligned}
$$

where $M$ does not depend on $r$. From $g \in C([0, T] ; E)$ it follows that $\underline{g}(\cdot, r)$, $\bar{g}(\cdot, r)$ are equicontinuous with respect to $r$, hence the convergences

$$
\left\|P_{N} \bar{g}(\cdot, r)-\bar{g}(\cdot, r)\right\| \rightarrow 0 \text { and }\left\|P_{N} \underline{g}(\cdot, r)-\underline{g}(\cdot, r)\right\| \rightarrow 0 \text { as } N \rightarrow \infty
$$


are uniform in $r$. Consequently

$$
\sup _{t \in[0, T]} D\left(g_{N}(t), g(t)\right) \leq \sup _{0 \leq r \leq 1} \max \left\{\left\|\bar{g}_{N}-\bar{g}\right\|_{\infty},\left\|\underline{g}_{N}-\underline{g}\right\|_{\infty}\right\} \rightarrow 0 .
$$

If the assumptions of Lemma 3 are satisfied, then by Lemma 4 and Lemma 3 we get the error estimate

$$
\sup _{t \in[0, T]} D\left(g_{N}(t), g(t)\right) \leq \sup _{0 \leq r \leq 1} \max \left\{\left\|\bar{g}_{N}-\bar{g}\right\|_{\infty},\left\|\underline{g}_{N}-\underline{g}\right\|_{\infty}\right\} \leq M h^{2} .
$$

To get the convergence estimate for the collocation method with rectangular basis, we redefine $X_{N}=\operatorname{span}\left\{\psi_{n}, n=0, \ldots, N\right\}$ and $P_{N}$ is then the interpolation projector onto $X_{N}$ with interpolation nodes $\tau_{n}$. We use the following standard result for the error of piecewise constant interpolation.

Lemma 5. Let $v \in W^{1, \infty}(0, T)$. Then, for the error in piecewise constant interpolation there holds

$$
\left\|P_{N} v-v\right\|_{\infty} \leq \frac{1}{2} h\left\|v^{\prime}\right\|_{\infty}
$$

In addition to the usual convergence result for rectangular basis, we also present a result about superconvergence at the collocation nodes.

Theorem 7. Let $K \in C\left(D_{T}\right), f \in C([0, T] ; E)$. Assume $h \rightarrow 0$ as $N \rightarrow \infty$. Then for sufficiently large $N$ the approximate equation (4.5) has a unique solution $g_{N}$ which converges uniformly to the exact solution $g$ of equation (3.1). If the assumptions of Lemma 2 are satisfied then the error estimate

$$
\sup _{t \in[0, T]} D\left(g_{N}(t), g(t)\right) \leq C h
$$

holds, where $C$ is a constant not depending on $N$. Moreover if the assumptions of Lemma 3 are satisfied and $\tau_{k}=\frac{1}{2}\left(t_{k-1}+t_{k}\right), k=1, \ldots, N$ then error estimate at collocation nodes

$$
\max _{k=1, \ldots, N} D\left(g_{N}\left(\tau_{k}\right), g\left(\tau_{k}\right)\right) \leq C h^{2}
$$

holds, where $C$ is a constant not depending on $N$.

Proof. The proof of the first part is similar to the proof of Theorem 6 .

To prove the superconvergence, we subtract from equation (6.1) the projected equation $P_{N} u=P_{N} A u+P_{N} f$ :

$$
u_{N}-P_{N} u=P_{N} A\left(u_{N}-u\right)=P_{N} A\left(\left(u_{N}-P_{N} u\right)+\left(P_{N} u-u\right)\right) .
$$

So,

$$
u_{N}-P_{N} u=\left(I-P_{N} A\right)^{-1} P_{N} A\left(P_{N} u-u\right),
$$


where $\left(I-P_{N} A\right)^{-1}$ is a bounded operator in $X_{N}$. We have

$$
A\left(P_{N} u-u\right)=\left(\begin{array}{c}
\mathcal{K}_{+}\left(P_{N} \underline{g}-\underline{g}\right)-\mathcal{K}_{-}\left(P_{N} \bar{g}-\bar{g}\right) \\
-\mathcal{K}_{-}\left(P_{N} \underline{g}-\underline{g}\right)+\mathcal{K}_{+}\left(P_{N} \bar{g}-\bar{g}\right)
\end{array}\right) .
$$

Since applying $P_{N}$ to this result uses only the values at $\tau_{k}$, we estimate one element of this vector at $\tau_{k}$. The others are similar.

$$
\begin{aligned}
\mathcal{K}_{+} & \left(P_{N} \underline{g}-\underline{g}\right)\left(\tau_{k}\right)=\int_{0}^{\tau_{k}} K_{+}\left(s, \tau_{k}\right)\left(P_{N} \underline{g}(s)-\underline{g}(s)\right) d s \\
= & \sum_{i=1}^{k-1} \int_{t_{i-1}}^{t_{i}} K_{+}\left(s, \tau_{k}\right)\left(\underline{g}\left(\tau_{i}\right)-\underline{g}(s)\right) d s+\int_{t_{k-1}}^{\tau_{k}} K_{+}\left(s, \tau_{k}\right)\left(\underline{g}\left(\tau_{k}\right)-\underline{g}(s)\right) d s .
\end{aligned}
$$

Using Taylor expansion at $\tau_{i}$ in each subinterval $\left[t_{i-1}, t_{i}\right]$ we have

$$
\begin{aligned}
\sum_{i=1}^{k-1} \int_{t_{i-1}}^{t_{i}}\left(K_{+}\left(\tau_{i}, \tau_{k}\right)+O(h)\right) & \left(\left(\tau_{i}-s\right) \underline{g^{\prime}}\left(\tau_{i}\right)+O\left(h^{2}\right)\right) d s+O\left(h^{2}\right) \\
= & \sum_{i=1}^{k-1} K_{+}\left(\tau_{i}, \tau_{k}\right) \underline{g^{\prime}}\left(\tau_{i}\right) \int_{t_{i-1}}^{t_{i}}\left(\tau_{i}-s\right) d s+O\left(h^{2}\right) .
\end{aligned}
$$

Since $\tau_{i}=\frac{t_{i-1}+t_{i}}{2}$, the integrals are all zero, so we get the estimate $O\left(h^{2}\right)$. For other elements the calculation is the same. Since $\left(I-P_{N} A\right)^{-1}$ is bounded, then from equation (6.2) we get $\left\|u_{N}-P_{N} u\right\|=O\left(h^{2}\right)$. Now notice that all the constants in the estimates are either independent of $r$ or contain first and second derivatives of $\underline{g}, \bar{g}$ which are uniformly bounded with respect to $r$ by Lemmas 2 and 3. Hence we have

$$
\max _{k=1, \ldots, N} D\left(g_{N}\left(\tau_{k}\right), g\left(\tau_{k}\right)\right) \leq C h^{2} .
$$

Remark 3. In general one has to solve the equations for each $r \in[0,1]$. In special cases, when $f(t)$ is a triangular, trapezoidal or interval fuzzy number for $t \in[0, T]$, then the solution is still of the same type, and it is enough to solve the equations only for $r=0$ and $r=1$.

\section{Examples}

In this section we present some numerical results. We used the collocation method with triangular and rectangular bases to solve approximately four examples of fuzzy Volterra integral equations. In examples 1 and 3 the kernels are nonnegative, in examples 2 and 4 they change sign.

We used uniform mesh and took $N=5,10,20,40,80,160$. To estimate the error $\max _{t \in[0, T]} D\left(g_{N}(t), g(t)\right)$ we calculated $\max _{k=0, \ldots, 3 N} D\left(g_{N}\left(\tilde{t}_{k}\right), g\left(\tilde{t}_{k}\right)\right)$, where $\tilde{t}_{k}=\frac{k}{3 N}, k=0,1, \ldots, 3 N$. We also calculated the ratios of consecutive errors. If the convergence is of order $O\left(h^{2}\right)$ then the ratios should be approximately 4; if the convergence is $O(h)$ then the ratios should be 2 . 
Example 1. Consider the fuzzy Volterra integral equation (3.1) with

$$
\underline{f}(t, r)=\left(t^{3}-t^{6} / 5\right)\left(r^{2}+r\right), \quad \bar{f}(t, r)=\left(t^{3}-t^{6} / 5\right)\left(4-r^{3}-r\right)
$$

and the kernel $K(s, t)=s t, 0 \leq s \leq t \leq 1$. The exact solution is given by $g(t, r)=t^{3}\left(r^{2}+r\right), \bar{g}(t, r)=t^{3}\left(4-r^{3}-r\right)$. In this case both the kernel and the solution are smooth. The results are given in Table 1.

Table 1. Comparison of numerical results for Example 1.

\begin{tabular}{ccccccc}
\hline$N$ & error (triang.) & ratio & error (rectang.) & ratio & error at $\tau_{k}$ & ratio \\
\hline 5 & $1.2491 e-01$ & & 1.0591 & & $1.9792 e-02$ & \\
10 & $3.2793 e-02$ & 3.8091 & $5.6263 e-01$ & 1.8825 & $6.2641 e-03$ & 3.1595 \\
20 & $8.4129 e-03$ & 3.8979 & $2.9036 e-01$ & 1.9377 & $1.7567 e-03$ & 3.5658 \\
40 & $2.1316 e-03$ & 3.9467 & $1.4755 e-01$ & 1.9679 & $4.6492 e-04$ & 3.7786 \\
80 & $5.3656 e-04$ & 3.9727 & $7.4382 e-02$ & 1.9837 & $1.1958 e-04$ & 3.8881 \\
160 & $1.3460 e-04$ & 3.9862 & $3.7345 e-02$ & 1.9918 & $3.0320 e-05$ & 3.9437 \\
\hline
\end{tabular}

The errors given in the table are fuzzy distances between the approximate and the exact solutions.

We see that for triangular basis the convergence is of order $O\left(h^{2}\right)$. For rectangular basis the convergence is $O(h)$ but at collocation points the convergence is $O\left(h^{2}\right)$. In fact, when we have better convergence at collocation points, then using these values we can construct a better approximate solution as well.

Example 2. Consider the fuzzy Volterra integral equation (3.1) with

$$
\begin{aligned}
& \underline{f}(t, r)=t r- \begin{cases}\frac{t^{4}}{4}(1-2 t)^{3} r, & t \leq \frac{1}{2}, \\
\frac{1}{64}(1-2 t)^{3} r+\left(\frac{t^{4}}{4}-\frac{1}{64}\right)(1-2 t)^{3}(2-r), & t \geq \frac{1}{2} .\end{cases} \\
& \bar{f}(t, r)=t(2-r)- \begin{cases}\frac{t^{4}}{4}(1-2 t)^{3}(2-r), & t \leq \frac{1}{2}, \\
\frac{1}{64}(1-2 t)^{3}(2-r)+\left(\frac{t^{4}}{4}-\frac{1}{64}\right)(1-2 t)^{3} r, & t \geq \frac{1}{2} .\end{cases}
\end{aligned}
$$

The kernel is $K(s, t)=s^{2}(1-2 t)^{3}, 0 \leq s \leq t \leq 1$ with

$$
K_{+}(s, t)=\left\{\begin{array}{ll}
s^{2}(1-2 t)^{3}, & t \leq \frac{1}{2}, \\
0, & t>\frac{1}{2},
\end{array} \quad K_{-}(s, t)= \begin{cases}0, & t<\frac{1}{2}, \\
-s^{2}(1-2 t)^{3}, & t \geq \frac{1}{2}\end{cases}\right.
$$

and the exact solution is given by $\underline{g}(t, r)=t^{3} r, \bar{g}(t, r)=t^{3}(2-r)$. In this case the kernel changes sign on the line $t=1 / 2$, but two derivatives with respect to $t$ are also zero on this line, so the kernels $K_{+}$and $K_{-}$are smooth (they have discontinuous third derivatives). Theoretically the solution might also have discontinuous third derivatives, but instead in our case $f$ and $\bar{f}$ have discontinuous third derivatives which compensate the singularities in the solution.

The results are given in Table 2 .

Again we can see that the theoretical convergence rates coincide with the real convergence rates. 
Table 2. Comparison of numerical results for Example 2.

\begin{tabular}{ccccccc}
\hline$N$ & error (triang.) & ratio & error (rectang.) & ratio & error at $\tau_{k}$ & ratio \\
\hline 5 & $5.0752 e-02$ & & $5.4180 e-01$ & & $3.4160 e-03$ & \\
10 & $1.3088 e-02$ & 3.8778 & $2.8514 e-01$ & 1.9001 & $1.5224 e-03$ & 2.2438 \\
20 & $3.3231 e-03$ & 3.9384 & $1.4624 e-01$ & 1.9498 & $4.9619 e-04$ & 3.0682 \\
40 & $8.3757 e-04$ & 3.9676 & $7.4053 e-02$ & 1.9748 & $1.4086 e-04$ & 3.5226 \\
80 & $2.1029 e-04$ & 3.9830 & $3.7262 e-02$ & 1.9873 & $3.7476 e-05$ & 3.7587 \\
160 & $5.2687 e-05$ & 3.9912 & $1.8690 e-02$ & 1.9937 & $9.6618 e-06$ & 3.8787 \\
\hline
\end{tabular}

Example 3. This example is taken from [17], Example 4.2.

Consider the fuzzy Volterra integral equation with

$$
\underline{f}(t, r)=\left(1-t-t^{2} / 2\right) r, \quad \bar{f}(t, r)=\left(1-t-t^{2} / 2\right)(2-r) .
$$

The kernel is $K(s, t)=t-s, 0 \leq s \leq t \leq 1 / 2$, and the exact solution is given by $\underline{g}(t, r)=(1-\sinh \mathrm{t}) \mathrm{r}, \bar{g}(t, r)=(1-\sinh \mathrm{t})(2-\mathrm{r})$. We used $T=1 / 2$ here, because in $[0,1]$ the function $f$ is not a fuzzy function.

The results are given in Table 3. In this example neither $f$ nor $g$ is Hukuhara differentiable, but as emphasized before, we only need differentiability of $\underline{f}, \bar{f}$ and $\underline{g}, \bar{g}$ to get the convergence results.

Table 3. Comparison of numerical results for Example 3.

\begin{tabular}{ccccccc}
\hline$N$ & error (triang.) & ratio & error (rectang.) & ratio & error at $\tau_{k}$ & ratio \\
\hline 5 & $1.1106 e-03$ & & $1.1072 e-01$ & & $7.8944 e-04$ & \\
10 & $2.8822 e-04$ & 3.8532 & $5.5882 e-02$ & 1.9823 & $2.0931 e-05$ & 3.7716 \\
20 & $7.3339 e-05$ & 3.9300 & $2.8056 e-02$ & 1.9907 & $5.3851 e-05$ & 3.8869 \\
40 & $1.8493 e-05$ & 3.9658 & $1.4061 e-02$ & 1.9953 & $1.3655 e-05$ & 3.9437 \\
80 & $4.6429 e-06$ & 3.9831 & $7.0391 e-03$ & 1.9976 & $3.4378 e-06$ & 3.9719 \\
160 & $1.1632 e-06$ & 3.9916 & $3.5217 e-03$ & 1.9988 & $8.6249 e-07$ & 3.9860 \\
\hline
\end{tabular}

Example 4. Consider the fuzzy Volterra integral equation with

$$
\underline{f}(t, r)=\left(t^{3}-\frac{t^{5}}{320}\right) r-\frac{49 t^{5}}{320}(r-2), \quad \bar{f}(t, r)=\frac{49 t^{5}}{320} r-\left(t^{3}-\frac{t^{5}}{320}\right)(r-2)
$$

and the kernel $K(s, t)=t-2 s, 0 \leq s \leq t \leq 1$. The exact solution is given by $g(t, r)=t^{3} r, \bar{g}(t, r)=t^{3}(2-r)$. In this case there is a sign change of the kernel along the line $s=t / 2$. Since this line does not have any endpoints or intersection points with the line $s=t$ inside $[0,1]$, the solution is smooth.

The results are given in Table 4 . 
Table 4. Comparison of numerical results for Example 4.

\begin{tabular}{ccccccc}
\hline$N$ & error (triang.) & ratio & error (rectang.) & ratio & error at $\tau_{k}$ & ratio \\
\hline 5 & $1.7384 e-03$ & & $4.6071 e-01$ & & $6.6514 e-03$ & \\
10 & $4.5297 e-04$ & 3.8377 & $2.4240 e-01$ & 1.9006 & $2.0008 e-03$ & 3.3244 \\
20 & $1.1391 e-04$ & 3.9765 & $1.2432 e-01$ & 1.9498 & $5.4034 e-04$ & 3.7028 \\
40 & $2.8520 e-05$ & 3.9941 & $6.2951 e-02$ & 1.9749 & $1.4021 e-04$ & 3.8538 \\
80 & $7.1327 e-06$ & 3.9985 & $3.1675 e-02$ & 1.9874 & $3.5699 e-05$ & 3.9275 \\
160 & $1.7833 e-06$ & 3.9996 & $1.5887 e-02$ & 1.9937 & $9.0062 e-06$ & 3.9639 \\
\hline
\end{tabular}

\section{Conclusions}

In this paper, we proved a regularity result for solution of fuzzy Volterra integral equations. This is a new result, at least for kernels which may change sign. We proposed the collocation method with triangular and rectangular basis functions for solving these equations. Using the regularity result we estimated the order of convergence of the method. The numerical results confirm the theoretical convergence estimates and good performance. The advantage of these methods is simplicity of use and robustness, i.e. they do not require high regularity of the solution, only piecewise $C^{2}$ is enough. If the solution is not smooth, then many other methods are not applicable, especially those which use Taylor expansions or high order polynomials to approximate the solution. On the other hand, if the solution is very smooth (analytic) then it is possible to use methods with much higher convergence rates.

For future research, we will investigate the solution of fuzzy Volterra integral equations with arbitrary crisp weakly singular kernels.

\section{Acknowledgement}

This work was supported by institutional research funding IUT20-57 of the Estonian Ministry of Education and Research.

\section{References}

[1] S. Abbasbandy, E. Babolian and M. Alavi. Numerical method for solving linear Fredholm fuzzy integral equations of the second kind. Chaos, Solitons and Fractals, 31(1):138-146, 2007. https://doi.org/10.1016/j.chaos.2005.09.036.

[2] E. Babolian, H.S. Goghary and S. Abbasbandy. Numerical solution of linear Fredholm fuzzy integral equations of the second kind by Adomian method. Applied Mathematics and Computation, 161(3):733-744, 2005. https://doi.org/10.1016/j.amc.2003.12.071.

[3] B. Bede. Mathematics of Fuzzy Sets and Fuzzy Logic, volume 295 of Studies in Fuzziness and Soft Computing. Springer, 2013. https://doi.org/10.1007/978-3642-35221-8.

[4] A.M. Bica. Error estimation in the approximation of the solution of nonlinear fuzzy Fredholm integral equations. Information Sciences, 178(5):1279-1292, 2008. https://doi.org/10.1016/j.ins.2007.10.021. 
[5] H. Brunner. Collocation methods for Volterra integral and related functional differential equations, volume 15 of Cambridge Monographs on Applied and Computational Mathematics. Cambridge University Press, 2004. https://doi.org/10.1017/CBO9780511543234.

[6] H. Brunner. Volterra integral equations: an introduction to theory and applications, volume 30 of Cambridge Monographs on Applied and Computational Mathematics. Cambridge University Press, 2017.

[7] S.S.L. Chang and L.A. Zadeh. On fuzzy mapping and control. IEEE Transactions on Systems, Man and Cybernetics, 2:30-34, 1972. https://doi.org/10.1109/TSMC.1972.5408553.

[8] D. Dubois and H. Prade. Towards fuzzy differential calculus part 3: Differentiation. Fuzzy sets and systems, 8(3):225-233, 1982. https://doi.org/10.1016/S0165-0114(82)80001-8.

[9] M. Friedman, Ma Ming and A. Kandel. Numerical solutions of fuzzy differential and integral equations. Fuzzy sets and Systems, 106(1):35-48, 1999. https://doi.org/10.1016/S0165-0114(98)00355-8.

[10] J.R. Goetschel and W. Voxman. Elementary fuzzy calculus. Fuzzy sets and systems, 18(1):31-43, 1986. https://doi.org/10.1016/0165-0114(86)90026-6.

[11] O. Kaleva. Fuzzy differential equations. Fuzzy sets and systems, 24(3):301-317, 1987. https://doi.org/10.1016/0165-0114(87)90029-7.

[12] R. Kress. Linear integral equations, volume 82 of Applied Mathematical Sciences. Springer, 2014. https://doi.org/10.1007/978-1-4614-9593-2.

[13] M. Mosleh and M. Otadi. Solution of fuzzy Volterra integral equations in a Bernstein polynomial basis. Journal of Advances in Information Technology, 4(3):148-155, 2013. https://doi.org/10.4304/jait.4.3.148-155.

[14] J.Y. Park and H.K. Han. Existence and uniqueness theorem for a solution of fuzzy Volterra integral equations. Fuzzy Sets and Systems, 105(3):481-488, 1999. https://doi.org/10.1016/S0165-0114(97)00238-8.

[15] F. Saberidad, S.M. Karbassi and M. Heydari. Numerical solution of nonlinear fuzzy Volterra integral equations of the second kind for changing sign kernels. Soft Computing, 2018. https://doi.org/10.1007/s00500-018-3668-x.

[16] P.K. Sahu and S. Saha Ray. A new Bernoulli wavelet method for accurate solutions of nonlinear fuzzy Hammerstein-Volterra delay integral equations. Fuzzy Sets and Systems, 309:131-144, 2017. https://doi.org/10.1016/j.fss.2016.04.004.

[17] S. Salahshour and T. Allahviranloo. Application of fuzzy differential transform method for solving fuzzy Volterra integral equations. Applied Mathematical Modelling, 37(3):1016-1027, 2013. https://doi.org/10.1016/j.apm.2012.03.031.

[18] L.A. Zadeh. Fuzzy sets. Information and control, 8(3):338-353, 1965. https://doi.org/10.1016/S0019-9958(65)90241-X. 